\title{
Docker layer placement for on-demand provisioning of services on edge clouds
}

\author{
Piet Smet, Bart Dhoedt and Pieter Simoens \\ IDLab, Department of Information Technology \\ Ghent University - imec \\ Technologiepark 15, B-9052 Ghent, Belgium
}

\begin{abstract}
Driven by the increasing popularity of the microservice architecture, we see an increase in services with unknown demand pattern located in the edge network. Predeployed instances of such services would be idle most of the time, which is economically infeasible. Also, the finite storage capacity limits the amount of deployed instances we can offer. Instead, we present an on-demand deployment scheme using the Docker platform. In Docker, service images consist of layers, each layer adding specific functionality. This allows different services to reuse layers, avoiding cluttering the storages with redundant replicas. We propose a layer placement method which allows users to connect to a server, retrieve all necessary layers -possibly from multiple locations- and deploy an instance of the requested service within the desired response time.

We search for the best layer placement which maximizes the satisfied demand given the storage and delay constraints. We developed an iterative optimization heuristic which is less exhaustive by dividing the global problem in smaller subproblems. Our simulation results show that our heuristic is able to solve the problem with less system resources. Last, we present interesting use-cases to use this approach in real-life scenarios.
\end{abstract}

Keywords: service-centric, on-demand, placement algorithm, long-tail, services, docker

\section{INTRODUCTION}

Over the last few years the amount of services on the Internet has increased rapidly while users have increasing performance demands. Cloud Computing was developed to facilitate resource scaling, resilience and security while distributed clouds bring services to the edge network, closer to the users [1]. The monolithic nature of most services prevents different components of a service to be scaled separately, making high service availability difficult to accomplish under increasing demand. Instead of interweaving all functionality into one service, we see more and more services designed as separate, loosely coupled services which communicate with each other to accomplish full functionality. This approach is commonly referred to as microservice architecture [2] [3]. Microservice architectures allow efficient code reusability and more fine-grained scaling.

Another reason for the popularity of microservices is the emergence of container virtualization. Cloud Computing uses virtualization techniques to deploy, relocate or scale Virtual Machines (VM) dynamically to meet the changing service requirements. Due to the high resource demand of VMs on the host machine there was a need for a more lightweight approach aimed at hosting a specific application, which led to the development of software containers [4]. Unlike VMs, software containers share the same operating system kernel with the host machine to reduce the on-demand provisioning overhead and provide more efficient resource usage. This design goes hand in hand with microservice architectures as each lightweight service component can be provisioned ondemand in a container and scaled for its specific requirements.

Containerized services are increasingly deployed on edge cloud infrastructure, e.g. for low-latency processing of IoT sensor data or to protect sensitive data by keeping the analysis close to the source of the data [5].

These software containers must be deployed in a way that efficiently uses the limited resources available. Although there is already a large body of work on service placement for popular services based on the availability of user demand patterns, our focus is on the growing amount of long-tail services coming from the microservice architecture. We define a long-tail service as a service with infrequent demand, for which no statistically reliable demand pattern is available to find a suitable pre-deployment scheme. The predicate 'longtail' stems from the business marketing domain, and refers to the shape of the curve when ranking sold items in decreasing popularity [6]. While there are a few very popular products, the long tail of a very large majority of infrequently sold items (niche markets) represents an economically equality attractive business opportunity. With the advent of cloud computing, the Software-as-a-Service model has made it much easier to offer a delivered service to the end-user which resulted in a very long tail of services that are used relatively infrequently [7] [8]. Recently, the shift from monolithic application designs to micro-service oriented system architectures further increases the number of services that must be orchestrated. Note that a novel service might start as a long-tail service, but might lose this status as it becomes more popular and more data on demand patterns become available.

A key element in the long-tail paradigm is that the economic valorization of the items in the long tail should not be neglected. Hence, the long tail of software services should not be overlooked by operators of distributed cloud infrastructure.

When it comes to finding an optimal deployment scheme, pre-deploying service instances across the edge network is economically infeasible because they are infrequently used. Additionally, pre-deploying instances limits the number of services we can offer due to limited resources on edge nodes which is an issue for the high amount of services considered. 


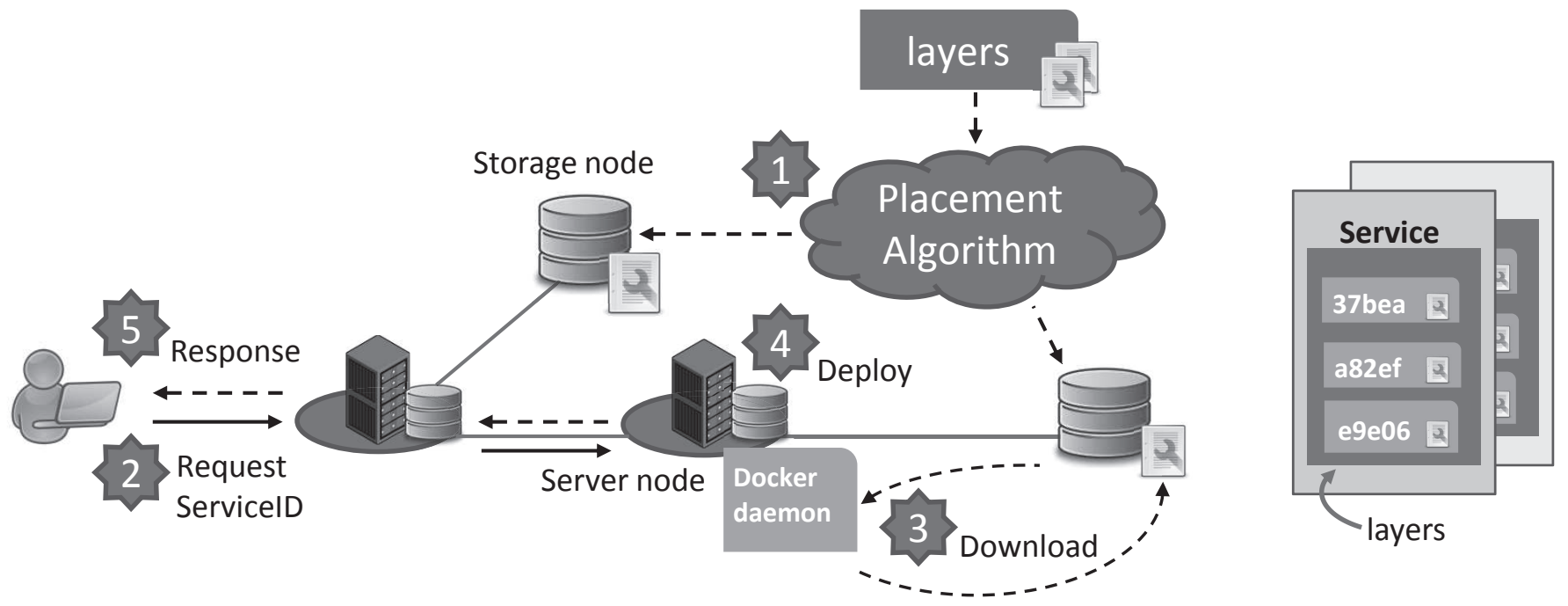

Fig. 1. We place service layers on storage nodes so that, upon service request, Docker can download the required service layers, possibly from different locations, and deploy a service instance within the desired response time. The server nodes, located in the edge network, have limited storage capacity.

In this case on-demand provisioning is a better solution than pre-deploying instances across the edge network. To facilitate on-demand provisioning we can use the popular framework Docker [9], an open-source project which utilizes operatingsystem-level virtualization to facilitate the deployment of applications inside software containers. Container images are broken down into layered filesystems (layers) which can be considered the service building blocks, each adding a piece of required functionality. Layers can be shared by multiple services and retrieved from remote storage nodes. This allows more efficient storage usage and adds new optimization possibilities to the service placement problem which have not been explored yet.

In this paper we propose a service placement method which maximizes the amount of clients we can serve on-demand within a desired service response time, focusing on services without known demand patterns. Following the Docker design, services are broken down into layers which can be shared by multiple services. Assuming that a client may connect from any given client location, we place the layers so that users can connect to a server, retrieve the layers from their perspective storage nodes and deploy a service instance on that server within a desired response time. Docker handles the layer retrieval and instantiation so we only concern ourselves with finding a suitable location for the layers (Fig. 1).

The research presented in this paper extends our previously published work from [10]. We added an additional approach to support parallel downloads as seen in the Docker v2 API. Additionally, we introduce an iterative search method to solve larger scenarios using less system resources. New benchmarks were added to compare our approach with existing solutions. Last, simulation results were obtained by modeling real-life networks and latency distributions.

In the remainder of this paper we present our service placement models and discuss our experiment results. In the next section we describe related work on service placement, multi- stage selection algorithms and relevant research on Docker, all important aspects of our research. In section III we explain the Docker software in more detail as it is an important part of our research. In section IV we formulate our problem statement for both a serial and parallel download model. Next, we introduce an iterative search method in section V. Our simulations are driven by realistic datasets so that our results match real-life scenarios, we describe this process and our results in section VII. Last, we discuss future work which we will expand on in section VIII.

\section{RELATED WORK}

1. Service placement. Due to dynamic pricing schemes on Clouds, service placement algorithms often aim to minimize the total hosting cost for a set of services while trying to maximize a performance metric [11]. In [12] the authors minimize the hosting cost while ensuring a minimum response time. This is also a dynamic algorithm which considers change in user demand over time and adjusts the amount of service instances deployed at each datacenter. The researchers assume that services are instantiated so users can connect instantly. In contrast, we are dealing with services without known demand patterns (e.g. long-tail services or newly deployed services) so we cannot assume any distribution of user demand (in most cases the service runs idle), making it economically infeasible to pre-deploy such instances. Moreover, the storage capacity constraints limit the amount of services we can deploy.

Similar to our model, other approaches aim to maximize the satisfied demand given a set of constraints (budget, storage capacity...). In [13] the authors assume that services are instantiated before a request arrives. The selection algorithm in this research only needs to connect clients to servers as it assumes the services is already fully pre-deployed. As this is economically infeasible for services with limited edge cloud resources, our approach places service layers on strategic 
locations so users can deploy services on-demand within a desired response time.

Other research on service placement in so called micro clouds has similar goals to ours; to make services easily accessible in the user's proximity. In [14] the authors find the optimal placement for services by considering bandwidth, network delay and node availability. This research was later optimized [15] with a focus on availability and bandwidth. Although bandwidth is only a secondary objective in our research, it affects the download time of our service layers which is part of our primary user satisfaction constraint and therefore also relevant.

The authors of [16] present a model that combines cost efficiency, bandwidth, computational resources and client location. Finding a solution for this model also requires an exhaustive search which is not suitable for large-scale scenarios. Similar to our research, an iterative approach is employed to get near-optimal results. However, this existing research also assumes a clear mapping from clients to service instances while we also consider the provisioning time to pull service layers from storage to server nodes before we can deploy an instance on-demand.

2. Multi-stage selection. To validate a placement, our solution must find the best possible selection to validate that users can be served on-demand with this placement. A practical example is shown in [17] where the authors aim to minimize construction costs by selecting a suitable crane for each supply demand and finding the best location for each crane at the same time. This is similar to the Facility Location problem which aims to find the optimal location, amount of facilities and client-facility selection to serve as many customers as possible within a certain response time. This is very useful to find the right location to place hospitals to cover as many potential locations within the desired time.

However, our solution not only assigns servers to clients but also needs to find the best storage location to download layers from to ensure that the instance can be deployed in the desired time. This is similar to the multi-stage supply chain problem where consumers are not only assigned to a distribution center but distribution centers are also linked to production plants. An example of the multi-stage supply chain is presented in [18] where the author attempts to find a distribution center for each client, as well as which factories deliver to which distribution center. Most related research on the multi-stage supply chain problem [19] [20] all face the same difference from our research; existing research typically assumes knowledge of the user demand patterns which are harder to predict for long-tail services and newly registered services. By solving the on-demand scenario in our research, users can come online from any location and deploy a service instance on-demand within the desired response time, without needing any knowledge of the user demand.

A recent study on the two-level uncapacitated facility location problem [21] also tackles this case without assuming specific demand patterns. Although our challenge is also a twolevel selection, our research is more challenging than selecting a server and storage for each client, as we must consider that all layers of a service can be located on different storages.
This means that a (client, server, storage) triplet in existing research now turns into multiple (client, server, storage, layer) quadruples. Additionally, our research does consider storage capacity and makes use of layer reusability to maximize satisfied demand given this limited capacity.

3. Docker in Fog computing. Fog computing, or also called edge computing, uses Internet of Things (IoT) devices to leverage resources in the edge network. Similar to how our research finds the optimal server and storage nodes to deploy Docker layers on, Fog computing attempts to make optimal use of nearby (storage, networking or computational) resources. Docker containers are becoming an increasingly popular choice for Fog computing development. Some examples of research relying on Docker virtualization in a Fog computing and IoT environments can be found in [22] and [23]. In the research presented in [24], a real-world Fog landscape was made using Docker containers to support the research.

IFogStor [25] introduces a data placement strategy focused on fog infrastructure. Similar to how our research attempts to quickly retrieve Docker layers in the right location, iFogStor attempts to reduce the data retrieval and service latency in a Fog infrastructure. Using integer programming and a search heuristic, this placement strategy managed to reduce the average latency by $60 \%$ compared to existing fog approaches.

\section{DOCKER}

Docker provides a lightweight alternative to full virtualization using Linux container (LXC) based operating-systemlevel virtualization. It provides portable deployment of software containers across platforms, shared and reusable layers and versioning amongst others. In comparison to VMs, Docker containers require less system resources, deploy in subseconds and focus on running applications rather than emulating hardware. Due to its modular design and ability to easily deploy and re-use services across platforms, Docker makes it easier to ensure the same deployment environment is used amongst researchers, thereby facilitating reproducible results.

Docker services are built from a series of layers, allowing us to store replicas on different locations and reuse these layers across different services. Our placement method considers these characteristics when finding a location to place layers so that a service instance can be deployed almost instantly upon request arrival. In our model each service consists of a set of reusable layers and a service can only be deployed when each layer is located on the same host.

In Docker, each service consists of a base layer and every consecutive layer adds a piece of functionality. With one pull command, Docker is able to retrieve closeby replicas of each layer and compile the service on the local host. The retrieval process differs depending on the version of Docker we use. In the first version, a service is defined by its top layer which contains a reference to its parent layer, the layer situated directly below it in the Docker image. By following the parent layer references, we eventually end up at the base layer, which means all layers of that service are retrieved and we can compile the service. Although each layer can be retrieved 
TABLE I

PROBLEM STATEMENT VARIABLES

\begin{tabular}{|l|l|}
\hline Parameter & Description \\
\hline $\mathrm{N}$ & The union of all client, server and storage nodes. \\
\hline $\mathrm{I}$ & The client node collection. \\
\hline $\mathrm{J}$ & The server node collection. \\
\hline $\mathrm{K}$ & The storage node collection. \\
\hline $\mathrm{S}$ & The service collection. \\
\hline $\mathrm{L}$ & The collection of layers. \\
\hline$L_{s}$ & The collection of layers $l$ in service $s$. \\
\hline$d_{i, j}$ & Minimal latency between node $i$ and $j$ \\
\hline$H_{i, j}$ & Minimal hop count between node $i$ and $j$ \\
\hline$D_{j, k, l}$ & Download time between node $j$ and node $k$ for layer $l$. \\
\hline$C_{k}$ & Storage capacity on storage node $k$. \\
\hline$M_{l}$ & Storage capacity required by layer $l$. \\
\hline$D_{s}$ & Maximum tolerable delay for service $s$ so clients will be satisfied. \\
\hline$T_{l}$ & Deployment time for layer $l$. \\
\hline$Y_{k, l}$ & DECISION VARIABLE: $Y_{k, l}=1$ if layer $l$ is downloadable from storage $k$, else zero. \\
\hline$P_{i, j, s, k, l}$ & DECISION VARIABLE: $P_{i, j, s, k, l}=1$ if client $i$ connects to server $j$ to run service $s$ and downloads layer $l$ from storage $k$. \\
\hline$Q_{i, j, s}$ & $\begin{array}{l}\text { DECISION VARIABLE: } Q_{i, j, s}=0 \text { (a logical solution) if the selection variable P assigned all layers of a service } s \text { to a client and that client } \\
\text { only connects to one server } j \text { for all layers of that service. Else } Q_{i, j, s}=1 \text { denotes an infeasible solution. }\end{array}$ \\
\hline$W_{i, s}$ & $\begin{array}{l}\text { DECISION VARIABLE: Parallel download time of server s for client i. This is the max download time of each layer in s through the } \\
\text { selected server and storages. (cfr. section IV-B) }\end{array}$ \\
\hline$Z_{k, l}$ & Cumulative placement result from previous runs in $S P S$ (cfr. section V) \\
\hline
\end{tabular}

from a different storage location, this version assumes a serial download model; we need to retrieve each layer at once to find all layers of that service.

Since each layer was not only defined by its functionality but also by its parent reference, multiple layers with the same function were not recognized as the same layer by Docker, essentially defeating the purpose of reusability.

In the second version of the Docker API, each service is identified by its functionality by using a hash of the layer as an identifier. This allowed Docker to reuse services with the same functionality across multiple services with different base layers. Moreover, the layered structures of popular services which are officially supported by Docker are known by the Docker daemon, allowing it to retrieve each layer in parallel as it no longer needs to discover the parent layer references. This substantially speeds up the layer retrieval process.

In our research we examine both the serial and parallel download model, described in the following section.

\section{SERVICE PLACEMENT}

Our goal is to maximize the amount of users we can serve within a desired response time (satisfied clients), focusing on services with unknown demand pattern (e.g. long-tail services and newly registered services). The response time includes the client-server latency, the time to pull all layers onto the server node and the time required to compile the layers into a service image and deploy a service instance. We focus on the edge cloud case where small processing servers with limited resources are located in the edge network, closer to the user, while large storage nodes are located deeper into the backbone network. Pre-deploying idle service instances is economically infeasible and limits the amount of services we can offer due to the limited server resources. Instead, we aim to maximize the users we can serve on-demand by placing Docker layers at strategic locations so users can connect to a server node, pull the necessary layers and deploy a service instance on that server node within the desired response time. Our solution focuses on finding the optimal layer placement and we use Docker to facilitate service deployment. If there is no layer placement that satisfies all clients then we find the solution which maximizes the amount of satisfied clients.

We formulate our problem for both a serial and parallel download model. The serial model assumes that all layers of a service must be downloaded one at a time, similar to Docker v1 where each layer contained a field that points to the next required layer in the service. The parallel model follows a Docker v2 approach where the service structure is known beforehand and all layers of a service can be downloaded simultaneously. In both models we aim to solve our objective, to maximize the satisfied users, while using constraints to ensure that our solution is feasible.

To reduce the amount of required system resources, we divide our larger datasets into smaller ones which include only one service of the original problem. We then solve each smaller dataset consecutively and remember our previous decisions to make use of shared layers between multiple services. We compare the performance of both methods and evaluate their scalability.

\section{A. Problem statement: Serial download}

Variables. Consider a set of clients $I$, servers $J$ and storage nodes $K$. We define the collection of network nodes $N=$ $I \cup J \cup K$ as the union of these three collections. The collection of services is $S$ and each service $s \in S$ consists of a set of layers $L_{s}$ required to deploy an instance of $s$. The collection of layers to be placed on storage nodes is $L$ but a layer $l \in L$ may be shared by multiple services and belong to more than one $L_{s}$.

The decision variables can take the values one or zero. $P_{i, j, s, k, l}=1$ if client $i$ connects to server node $j$ to pull layer 
$l \in L_{s}$ from storage node $k$, otherwise it is 0 . If we place layer $l$ on storage node $k$ then $Y_{k, l}=1$, otherwise 0 .

Constraints. The amount of used storage capacity cannot exceed the available storage capacity

$$
\sum_{l \in L} Y_{k, l} * M_{l} \leq C_{k} \forall k \in K
$$

where $C_{k}$ is the storage capacity of storage node $k \in K$ and the storage capacity required by layer $l$ is $M_{l}$. Each server node also has limited storage capacity to instantiate services or pre-deploy layers on, which is modeled as an additional storage node with latency zero to that server node.

A client $i$ can only pull layer $l$ from storage node $k$ to server node $j$ if layer $l$ is placed on storage node $k$

$$
P_{i, j, s, k, l} \leq Y_{k, l} \forall i, j, s, k, l
$$

For each request for service $s$ from client $i$, our selection result $P$ must assign exactly one server node $j$ where the client must connect to and for each layer $l$ in service $s$ there must be exactly one storage node $k$ where server node $j$ pulls layer $l$ from. If there were multiple options, we could select multiple duplicates from different locations, which is undesirable.

$$
\sum_{j \in J} \sum_{k \in K} P_{i, j, s, k, l} \leq 1 \forall i, s, l \in L_{s}
$$

Any selection we make needs to be valid; if the selection variable $P_{i, j, s, k, l}$ does not select a storage location for every layer $l$ from service $s$ for client $i$, then that client can never be satisfied for that service. Therefore, when our selection result $P_{i, j, s, k, l}$ assigns a client $i$ to a server node $j$ for service $s$, then it must also assign a storage node $k$ for each layer $l \in L_{s}$ where node $j$ can download that layer from. We define the decision variable $Q_{i, j, s}$ to mark a selection as valid $\left(Q_{i, j, s}=\right.$ 0 ) when all the layers of a service are selected in $P_{i, j, s, k, l}$, or invalid $\left(Q_{i, j, s}=1\right)$ if not all layers of a service are assigned in $P_{i, j, s, k, l}$. This can be written as

$$
Q_{i, j, s} \geq \frac{L_{s}-\sum_{k \in K} \sum_{l \in L_{s}} P_{i, j, s, k, l}}{L_{s}} \forall i, j, s
$$

Last, the selection must allow a client $i$ to connect to a server $j$, pull layers $l \in L_{s}$ from storage node $k$ and deploy them on server node $j$ within the desired response time $D_{s}$ for service $s$

$\sum_{j \in J} \sum_{k \in K} \sum_{l \in L_{s}} P_{i, j, s, k, l} *\left(\frac{d_{i, j}}{s i z e\left(L_{s}\right)}+D_{j, k, l}+T_{l}\right) \leq D_{S} \forall i, s$

We define the latency between two nodes $i, j \in N$ as $d_{i, j}$ and the time to download layer $l$ from storage node $k$ to server $j$ as $D_{j, k, l} . T_{l}$ is the required time to install a layer on the server after downloading it. To avoid double summation of the clientserver latency in the nested sum, we divide the latency by the amount of layers in $L_{s}$ as the client only connects to the server once for each service request.

Objective function. We aim to find the placement values $Y_{k, l}$ and selection values $P_{i, j, s, k, l}$ which maximize the amount of satisfied users. Any selection where $Q_{i, j, s}$ is not zero will result in unsatisfied clients. When we find a solution where $Q_{i, j, s}$ is zero and the solution is within the boundaries of all our other constraints, then a user is satisfied. Therefore, by searching for the solutions where $Q_{i, j, s}$ is zero, within the boundaries of our constraints, we find the placement and selection with the maximum amount of satisfied clients. In other words, we need to minimize $Q_{i, j, s}$ :

$$
\text { minimize } \sum_{i \in I} \sum_{j \in J} \sum_{s \in S} Q_{i, j, s}
$$

Note that Eq. 3 guarantees that only one server node $j$ will be selected for each client-service pair.

If multiple solutions can satisfy the same amount of users, we search for the solution that minimizes the used capacity. The resulting objective function is:

$$
\text { minimize } \frac{\sum_{k \in K} \sum_{l \in L} Y_{k, l}}{|K| .|L|}+\sum_{i \in I} \sum_{j \in J} \sum_{s \in S} Q_{i, j, s}
$$

where $|\mathrm{K}|$ is the amount of elements in $\mathrm{K}$ and $|\mathrm{L}|$ the amount of elements in L. When multiple solutions result in the same satisfied demand, the first term will be a tiebreaker that selects the solution with the least capacity used. We will always prioritize the satisfied demand as the minimum unit of the second term is one, while the first term is always smaller than one because we divided by $|\mathrm{K}|$.|L|.

We use integer linear programming (ILP) [26] to solve the above problem statement using CPLEX. As we place layers for all services in this method and assume a serial download model, we call this approach the All Services Serial ILP (ILP$A S-S)$. In the next section we describe how to apply this approach to a parallel download model.

\section{B. Parallel model}

The previous problem statement assumes that all layers are downloaded sequentially which is the case in the Docker v1 API where each layer referenced the next layer to be downloaded (parent layer). However, Docker v2 introduced parallel layer downloads so all layers can now be fetched simultaneously. This limits the influence of storage node locations and puts more importance on network latency between clients and servers. We modify our problem statement from the previous section to reflect this change.

To adjust the serial download model to a parallel model we must replace the sum of all layer download times $D_{j, k, l}$ in Eq. 5 with the maximum download time of all layers in the service we are looking to deploy. To linearize this maximization function, we extend our model in two ways.

First, we introduce a supporting decision variable $W_{i, s}$ as the maximum download time of all layers of service $s$ when downloaded from the selected server and storage nodes for client $i$ as determined by $P_{i, j, s, k, l}$.

Second, in Eq. 5 we change the sum of layer download times with the one-time parallel download time $W_{i, s}$, as defined above 
$W_{i, s}+\sum_{j \in J} \sum_{k \in K} \sum_{l \in L_{s}} P_{i, j, s, k, l} *\left(\frac{d_{i, j}}{\operatorname{size}\left(L_{s}\right)}+T_{l}\right) \leq D_{S} \forall i, s$

Then we ensure that $W_{i, s}$ is equal or larger than the actual maximum download time of all layers in the requested service $s$ for client $i$

$$
W_{i, s} \geq P_{i, j, s, k, l} * D_{j, k, l} \forall i, j, k, s, l \in L_{s}
$$

Since the solver is trying to maximize satisfied demand, it will assign small values to $W_{i, s}$ so that the total download time is smaller than the allowed delay (cfr. Eq. 8). However, by definition, $W_{i, s}$ will still be larger than the longest download time for any layer from the selected server. As a result, $W_{i, s}$ is a good representation for the maximum download time of all the layers in that service. Therefore, using $W_{i, s}$ in Eq. 8 allows us to model a parallel download time rather than the serial download time from Eq. 5.

This is the parallel model of ILP-AS (ILP-AS-P), while section IV-A assumed a serial download model (ILP-AS-S).

\section{ITERATIVE SEARCH}

Whether we use the serial or parallel model, scalability remains a challenge for ILP solvers. Typically, these solvers require large amounts of system resources to solve the problem statement and take a very long time to run. We can reduce the dataset by combining smaller layers in a service to one larger layer or by combining similar services. However, our experiments [10] showed that these methods are not impactful enough to allow ILP-AS (cfr. section IV-A) to solve very large problems.

The next step in our research was to find search heuristics to find a layer placement without requiring large amounts of system resources like $I L P-A S$. A first attempt came from a top down approach; the search heuristic places each layer on every storage node and then removes one layer at a time until the available storage capacity is sufficient for the remaining layers. Each iteration we removed the layer which least affected the satisfied demand in an attempt to keep the satisfied demand maximized. Our second heuristic was a bottom up approach were each layer is placed, one at a time, each time selecting the layer and storage node which adds most to the satisfied demand. However, both approaches are greedy and experiments showed that they performed considerably worse than ILP-AS, even on smaller scale scenarios. Additionally, each step of these approaches required an exhaustive search, making it less feasible for large scale scenarios due to the increasing computation time.

Rather than utilizing a greedy heuristic, we attempted to solve the layer placement problem with the Simulated Annealing search heuristic. Starting with a randomized solution, we make small changes to the current solution and compare both placements. To avoid getting stuck in a local optimum, the heuristic sometimes continues with a worse solution than the current best to try and explore other areas of the search space. In the end, we utilize the layer placement which resulted in the highest satisfied demand. Although more promising than our greedy top down or bottom up approaches, this algorithm also failed to be a convincing competitor for ILP-AS.

Instead, we solve the problem by placing layers for one service at a time. We employ a similar model as described in section IV-A and run it iteratively to deploy one service at a time, where each iteration deploys the next service and considers the placement decisions from the previous iterations. We repeat this until we notice that additional iterations do not increase the satisfied demand level. We call this method Sequential Per Service (SPS), as we now place one service per run and we repeat this process for each service. This method can be applied to both the serial $(S P S-S)$ and parallel (SPS-P) ILP models described in the previous sections.

Consider the following example; We need to serve 2 clients (U1, U2) for service A with 2 layers L1, L2 and B with layers L2, L3 on a network with two server nodes (J1, J2) and two storage nodes (K1, K2). Using ILP-AS, the solver evaluates every acceptable combination and comes to the conclusion that e.g. (L1, K1), (L2, K2) and (L3,K1) is the best placement and that both users $\mathrm{U} 1$ and $\mathrm{U} 2$ should connect to server node J1. Using SPS, the solver first attempts to place service A and finds (L1, K1) and $(\mathrm{L} 2, \mathrm{~K} 2)$ as best solution to maximize satisfied demand for service A. In the next iteration, the solver places service B and detects that layer L2 has already been placed on storage K2. The solver evaluates the best position for $\mathrm{L} 3$ and finds $(\mathrm{L} 3, \mathrm{~K} 1)$ as the optimal solution for service B if both clients $\mathrm{U} 1$ and $\mathrm{U} 2$ connect to server $\mathrm{J} 1$.

The final placement matches this of ILP-AS but each iteration of the SPS solver had less combinations to explore and reduced the search space by detecting previous placements. The disadvantage of SPS is that each iteration does not have any information about the next service to be placed, making it a greedy approach in worst case. In our example, it was possible for the first iteration of SPS to place L2 on storage K1, preventing the second iteration from ever finding the optimal placement from ILP-AS.

In section VII-C we evaluate the importance of the service order when placing services one at a time.

We start by introducing a new input variable called $Z_{k, l}$ which contains the result of the decision variable $Y_{k, l}$ of all previous services combined. During the first run $Z_{k, l}$ is an array of zeros and the solver attempts to place all layers of the first service so that the amount of satisfied demand for that service is maximized. In the second run the solver attempts to solve the problem statement with only the second service as input but this time with $Z_{k, l}$ containing the $Y_{k, l}$ values of the first run. The available capacity given as input for the second run is the original storage capacity minus the capacity used during the previous runs of our solver.

If $Z_{k, l}=1$ we already placed layer $l$ on storage node $k$ during one of the previous runs and there is no use of predeploying a second replica on that storage node. We ensure that this does not happen by forcing $Y_{k, l}$ to be zero if $Z_{k, l}=1$

$$
Y_{k, l} \leq\left(1-Z_{k, l}\right) \forall k, l
$$


This ensures that we make use of shared layers amongst multiple services.

We adjust Eq. 2 so $P_{i, j, s, k, l}$ can select a layer placed by $Y_{k, l}==1$ or a layer from one of the previous placements if $Z_{k, l}==1$

$$
P_{i, j, s, k, l} \leq Y_{k, l}+Z_{k, l} \forall i, j, s, k, l
$$

When finding the optimal placement for all services at the same time, our solver uses the available resources to maximize the satisfied demand for all services. However, when solving the problem one service at a time, our solver attempts to maximize the satisfied demand for each service in a greedy manner. The tiebreaker in our objective function (Eq. 7) minimizes the amount of layers placed, which prevents one iteration from using all the available storage locations and starving the services in upcoming iterations.

However, our objective function alone is not enough as one service is still allowed to place many layer replicas in order to achieve $100 \%$ satisfied demand for that service, while from a global point of view this means we are still starving the available resources for all other services. Therefore, we add an additional constraint limiting our problem to just one layer replica for each service we are deploying. If we have two services with one shared layer, each layer will be placed once besides the shared layer which is allowed to be placed twice. Instead of one replica per run we can also evaluate two, three ... replicas, which results in the following constraint

$$
\sum_{k \in K} Y_{k, l} \leq x \forall l \in L
$$

where $x$ denotes the amount of replicas for layer $l \in L_{s}$ we are allowed to place each time we deploy a service instance of $s$.

The objective function remains the same as in Eq. 7.

Using this adjusted model we place services one at a time and keep repeating this until we notice that placing additional services no longer increases the satisfied demand. In the next section we present our simulation results as we evaluate if this sequential approach makes our solution more scalable.

\section{BENCHMARKS}

Both of the suggested approaches in our research use an ILP solver (CPLEX) to solve the selection and placement problem. To verify our results, we compare with two existing approaches and implemented the following two benchmarks:

Random Allocation $(R A)$. We attempt to place each layer $l \in L$ on a randomly chosen storage location. We repeat this until all storage capacity is fully occupied and no additional layers can be placed. For each client and each service, we then select the server and storage locations which result in the fastest on-demand deployment time for that service and that client. This guarantees that each client selected the ideal locations for its specific request and no quicker access to that service is possible given the randomized placement. Last, we remove all layers which were not selected by any clients in the previous step to free up storage capacity for future use.
Greedy Allocation ( $G A)$. This benchmark is an adapted implementation of the 'Greedy Heuristic' presented in [27]. In this research, the algorithm starts by sorting a list of services by demand. As we are assuming that there is no knowledge of demand patterns, we start by randomly sorting the list of services instead. The remaining steps are:

1) Select the next service $s$ in the sorted list of services.

2) For each client $i \in I$, create a list of possible candidate storages to host an instance of service $s$ for it. Only include storages that have sufficient storage capacity available to host service s and a low enough delay.

3) Sort the storages from most to least possible clients (the storage which occurs on the most candidate lists comes first). Also include clients of previously placed services in this count. Sort the clients from least to most candidate servers (this will also give clients with only few possible candidates a chance).

4) Select the next storage $k$ in the sorted list.

5) For each client $i$ in the sorted list for which no candidate has been selected yet. Check if $k$ has enough remaining storage capacity and if there is a server $j$ which connects client $c$ and storage $k$ with low enough delay (take into account resources used by previously placed clients for service $s$ now). If any clients remain without candidate and not all servers have been checked yet, return to step 4.

6) If any services remain, goto step 1, otherwise finish.

The main drawback of this greedy approach is that this model does not consider separate layers and only speaks of services. As a result, in our implementation this means that all layers of one service will be placed on the same storage node. This is a general issue with existing research which is not modeled to Docker where each service consists of layered building blocks.

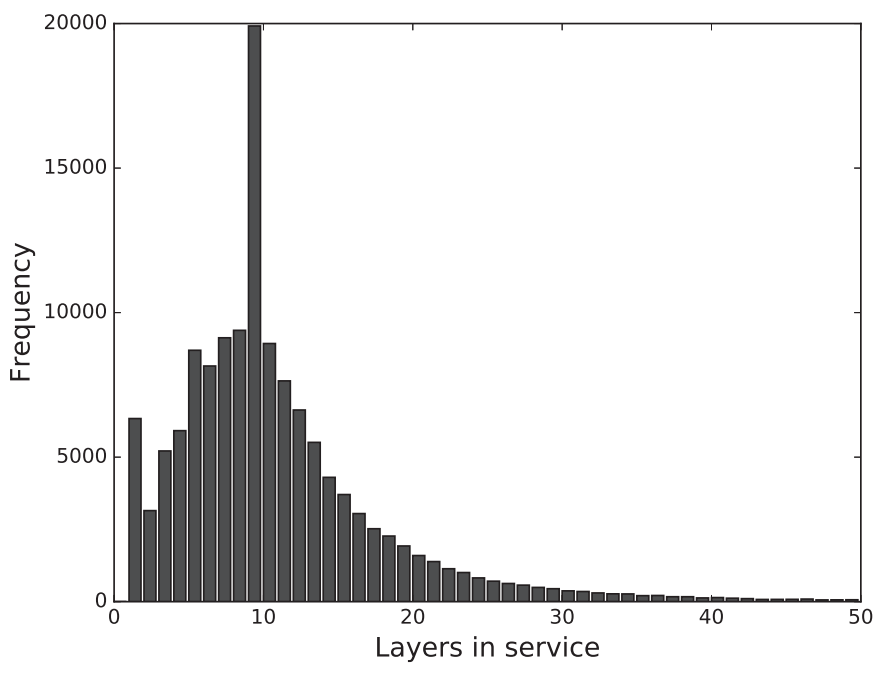

Fig. 2. A histogram visualizing the average layer distribution in Docker services. The $\mathrm{X}$-axis represents the amount of layers and the Y-axis indicates the frequency of services with this amount of layers. We observe that each service has 10 layers on average. 


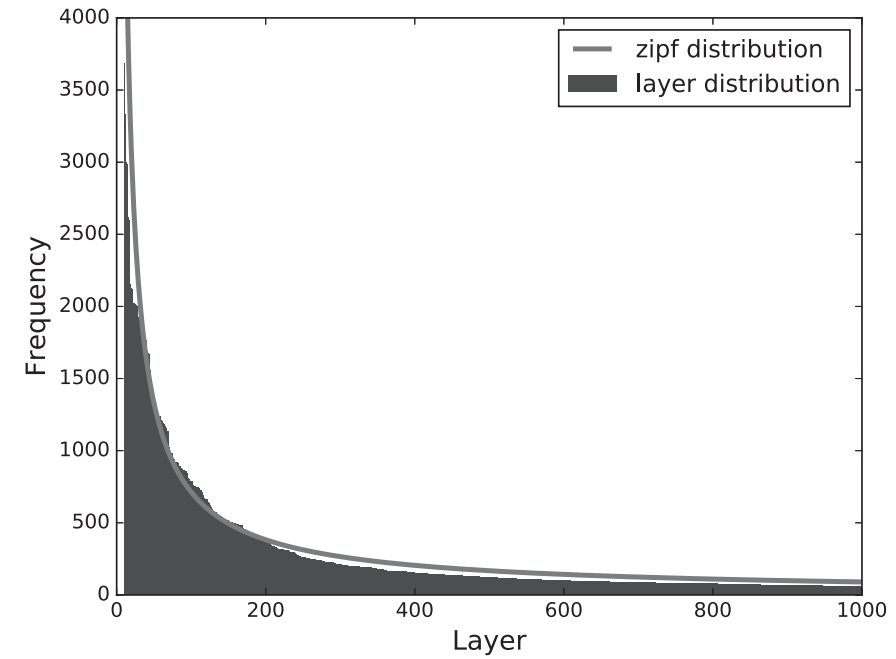

(a)

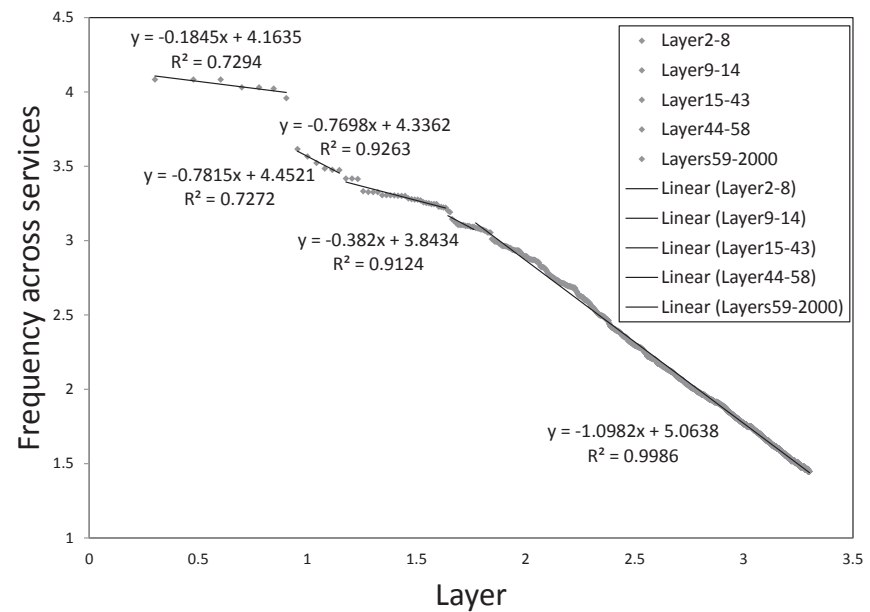

(b)

Fig. 3. Service layer reusability modeled as a Zipf distribution (a) and a sum of Zipf distributions in different ranges (b). These results show how often a specific layer is used in the different Docker services we crawled from the Docker repository. We can approximate this reusability behavior with a Zipf distribution or more accurately using a sum of Zipf distributions.

\section{SIMULATION RESULTS}

In this section we present our simulation results on the performance of our model described in section IV-A. We compare ILP-AS with our improved scalability efforts of SPS. Rather than solving the problem as a whole, we divide it into smaller pieces and solve each part iteratively, considering the decisions made during previous steps. For smaller problems our simulation results show that SPS achieves the same result as $I L P-A S$. This makes $S P S$ a viable approach to tackle larger scale problems which ILP-AS cannot solve without more system resources and execution time.

We also investigate the influence of the execution time on the quality of our solution. More specifically, how much performance do we gain from running the ILP-AS solver for a longer duration. A deployment solution found by the solver will remain a suitable solution as long as the amount of services and available resources stays the same. However, as services and user demand have a dynamic nature, it is in our best interest to keep the execution time as low as possible so we can react to changing dynamics. Although the latter is part of future work, we still touch on the performance gained from longer execution times.

The service characteristics were calculated from real-life Docker repositories as explained in section VII-A while our network topology is based on real-life latency distributions as described in section VII-B. All results are obtained by running each simulation on five different sample networks, each one generated based on these real-life parameters, and averaging the results. All simulations are performed on the iLab.t Virtual Wall [28] using a server with a Hexacore Intel E5645 (2.4GHz) CPU, 24GB RAM, 1x 250GB hard disk and $1-5$ gigabit network interface cards.

\section{A. Docker data crawling}

In order for our simulation results to match real-life applications, we crawled the Docker Hub [29] to determine the characteristics of Docker images. Inspecting the thousands of services in the Docker Hub showed us that services consist of few larger base layers and several smaller databases, including layers which only contain meta-data. On average, a service has 10 layers of which $10-20 \%$ are shared with other services. In Fig. 2 we illustrated our findings with a histogram, showing the amount of layers on the $\mathrm{X}$-axis and the frequency of services with this amount of layers on the Y-axis.

We plotted the layer distribution for all services in the Docker repository (Fig. 3 a). The $x$-axis shows the 1000 most frequently used layers in descending order. The y-axis indicates how many services used this layer. At first sight this resembles a Zipf distribution but a closer fit on a LOG-LOG plot reveals that we are dealing with a sum of Zipf distribution in different ranges, as shown in Fig. 3 b.

To ensure that our data model matches a realistic Docker model, we use this data to model the average layers per service, as well as the layer sizes.

\section{B. Latency distributions}

In order to compare our results with real-life applications, we need realistic latency distributions for our simulations. In this section we describe the latency models used in our experiments to describe a real-life network.

As discussed in section IV, our research focuses on the edge cloud case where services run in the edge network close to the users, while our data is stored on larger storage nodes located in the backbone network. Our simulation expects client-server latencies and the estimated time required to download a layer from a storage to a given server node. Considering that servers can make use of layers deployed on other servers, we observe three latencies to model:

1) The client-to-server latency. Each client must connect to an edge cloud where the service will run. The main factor in this delay is the time to reach the access point, usually done through the wireless network in modern scenarios. Research 


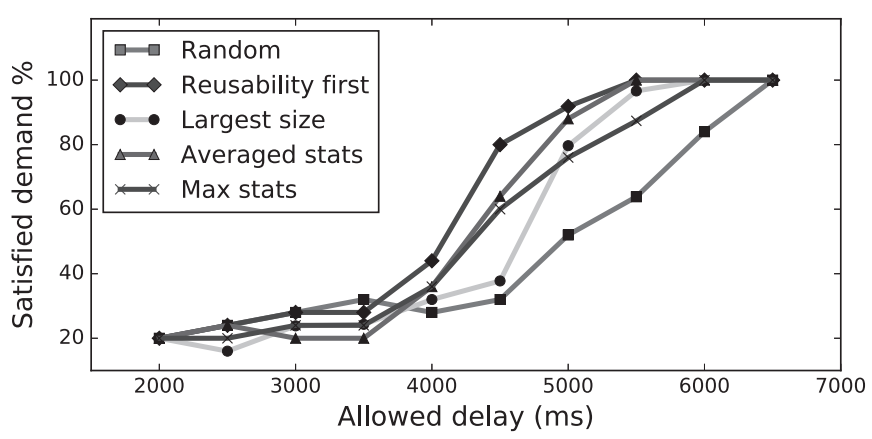

(a)

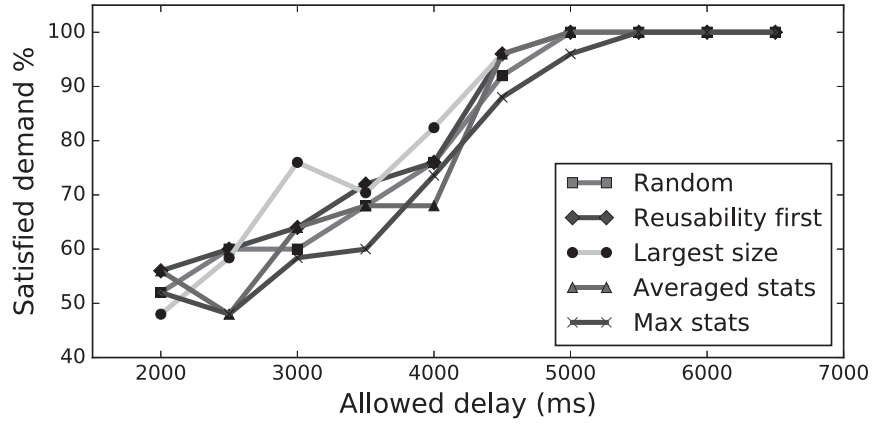

(b)

Fig. 4. The performance difference between different sorting methods in our heuristic approach for (a) 25 client nodes, 25 services, 2 server and 5 storage nodes and (b) 5 client nodes, 25 services, 5 server and 5 storage nodes. In both setups $20 \%$ of the service layers are reused by multiple services. We observe that the optimal sorting order depends on the experiment setup.

[30] shows that this latency is located between $15-60 \mathrm{~ms}$, while studies [31] on the latency to the provider of a $4 \mathrm{G}$ wireless network in the UK is close to 50ms. Therefore we use a uniform distribution between $30-70 \mathrm{~ms}$ to model the clientserver latency.

2) Server-to-server latency. Edge clouds can download layers from other nearby clouds in the edge network so we do not have to fetch all necessary data from far away storage nodes in the backbone network. Research [32] on the distance between two servers shows that each $1000 \mathrm{~km}$ adds $20 \mathrm{~ms}$ delay with a minimum threshold of $20 \mathrm{~ms}$ RTT. As our edge clouds are all located nearby in the edge network, we model this latency as a uniform distribution between 10 and $30 \mathrm{~ms}$.

3) Server-to-storage latency. When there is no copy of a desired layer available nearby, we must retrieve that layer from storage nodes located in the backbone network. These storage nodes are often spread out across the network and can even span continents. Sources [33] show that the average latency between storage nodes in the same continent is between 50$100 \mathrm{~ms}$, which is the distribution we chose to model the serverto-storage latency.

Using these link latencies we can calculate the estimated download time for each layer $\left(D_{j, k, l}\right)$ as layer size (in bits) divided by the link download speed (Gbps).

This latency distribution together with the Docker distribution is used as input to drive our simulations.

\section{Heuristic optimization}

In this section we investigate the importance of the service order in our iterative search method SPS. As there is a limited storage capacity on each node, not all services can be placed on the same node. The sorting order will dictate which services are prioritized and placed first.

Our search heuristic SPS reduces the required system resources by solving the problem one service at a time while utilizing previous decisions. We investigated the impact of the service sorting order on the performance of this sequential approach. As we focus on long-tail and newly registered services, we cannot predict the demand patterns for each service. From our crawling results (cfr. section VII-A) we know each layer in a service and their file sizes. We call the amount of services reusing a specific layer the reusability of that layer, assuming a layer can only occur once in each service. Using this information, we investigate the following four sorting methods:

1. Random: we traverse all services to be placed in a randomized order. This rather naive approach is used as a benchmark for our other methods.

2. Reusability first: in this method we prioritize layers which are reused by most services. By processing these layers first, they can be placed in the most centralized locations, allowing the maximum number of services to reuse that replica and thus saving storage capacity elsewhere. Therefore, we summarize the reusability frequency of all layers in a service and assign that as score to that service. We then solve SPS in order of decreasing service score, allowing services with the most frequently reused layers to be processed first.

3. Averaged stats: in the previous method it is possible that the sorted queue is dominated by tiny service layers which are reused frequently. As the server nodes in the edge network typically have less storage capacity than the large storage nodes in the backbone network, it may be beneficial for the heuristic to prioritize larger layers to take up the centralized locations. As we still want to consider the reusability of each layer, we now take a weighted average of both the reusability frequency and the layer sizes.

Layer score $=($ reusability $/$ average reusability $)+($ layer size / average size)

Service score $=\operatorname{sum}($ layer scores in service $)$

4. Max stats: A slight variation of the previous method where we normalize the factors with the maximum instead of the average. Our layer score now becomes:

Layer score $=($ reusability $/ \max$ reusability $)+($ layer size $/$ $\max$ size)

5. Largest size first: we attempt to prioritize larger base layers by sorting the layers by decreasing size. This will put base layers near the users in smaller storage nodes while the smaller remaining layers will be placed in larger but more distant storage nodes.

Fig. 4 shows the performance of our solution for each sorting method discussed above. The $\mathrm{X}$-axis represents the allowed response time to satisfy a client and the Y-axis 
represents the percentage of satisfied clients for the desired response time. In the first setup (Fig. 4 a) we have 25 client nodes, 25 services, 2 server and 5 storage nodes. Our second setup (Fig. 4 b) contains only 5 clients but 5 server nodes. In Fig. 4 a, reusability stands out as the best performing sorting method while random sorting is the least efficient method, taking the longest time to find a solution with $100 \%$ satisfied demand. However, in Fig. 4 b the different approaches have more server locations to choose from and we observe that our Max stats curve now performs worse than the other sorting methods.

After repeating the same experiment for different setups we concluded that the most suitable sorting order depends on the experiment setup. However, on average the sorting order does not have a big impact on the percentage of satisfied users.

In the next sections we use the reusability first method to sort services for SPS as this method was consistently amongst the best methods for different setups.

TABLE II

SIMULATION VARIABLES

All values marked with * are obtained through measurements of real-life systems. For other (artificial) variables only their relative values are

\begin{tabular}{cc} 
important. & Value \\
\hline Description & $200 \mathrm{MB}$ \\
\hline Lhe average size of a service* & 15 \\
\hline Layers per service* & $20 \%$ \\
\hline Layers shared by multiple services* & $2 \mathrm{~GB}$ \\
\hline Available storage node capacity & $600 \mathrm{MB}$ \\
\hline Available server node capacity & Uniform [30,70] ms \\
\hline Client-server latency* & Uniform $[10,30] \mathrm{ms}$ \\
\hline Server-to-server latency* & Uniform $[50,100] \mathrm{ms}$ \\
\hline Server-to-storage latency* &
\end{tabular}

\section{Execution time}

Given infinite system resources and time, ILP-AS will find an optimal solution to our placement problem. In this section we evaluate how much performance is lost by introducing a limited execution time, which is a more practical scenario. Additionally, we perform the same steps with SPS and compare the performance to ILP-AS. Our goal is to evaluate if the divide and conquer approach of SPS is beneficial given a limited execution time.

Our research focuses on solving a placement and selection problem using an ILP solver (CPLEX). We differentiate two techniques; the first method solves the entire problem in one run $(I L P-A S)$. The second method attempts to solve our problem for one service at a time (SPS), keeping in mind placement decisions from previous runs. Both approaches must evaluate possible placements by finding the optimal selection and calculating the satisfied demand.

The ILP-AS solver has a global view of our problem, including all layers to be placed, the possible storage locations and servers where clients can connect from. As a result, the solver will always find the optimal solution for any given scenario, assuming the system we run it on has sufficient memory. SPS solves the same problem but separately for each service, without considering the services that still need to be placed and which layers they have in common with other services. Therefore, the solution of SPS will be the same as ILP-AS at best.

Our experiments showed that, for small scenarios, SPS is close to the optimal solution found by ILP-AS but it does not always guarantee that this solution can be found, as explained in the example in section V. For smaller scenarios where ILP$A S$ is able to solve the problem in a feasible time span, ILP$A S$ is always the recommended approach as it guarantees an optimal solution.

However, due to the complexity of the ILP-AS model, this approach scales poorly when the size of our problem increases. The system will lack the memory required to solve the problem or require a much longer execution time to find a solution. SPS only considers one service per run, reducing the amount of memory required in each run of our solver. As a result, this approach is able to find better solutions than $I L P-A S$ given a limited execution time or system memory.

Our goal in this paper is to demonstrate that $S P S$ can achieve better results than $I L P-A S$ given a limited execution time, due to less system memory consumption in each run. As ILP-AS is not able to solve larger scenarios in a feasible time, we forcefully interrupt the $I L P$-AS solver after a certain execution time and retrieve the current best solution. We argue that, in the same time span, SPS will find a current best solution which satisfies more users due to solving much faster.

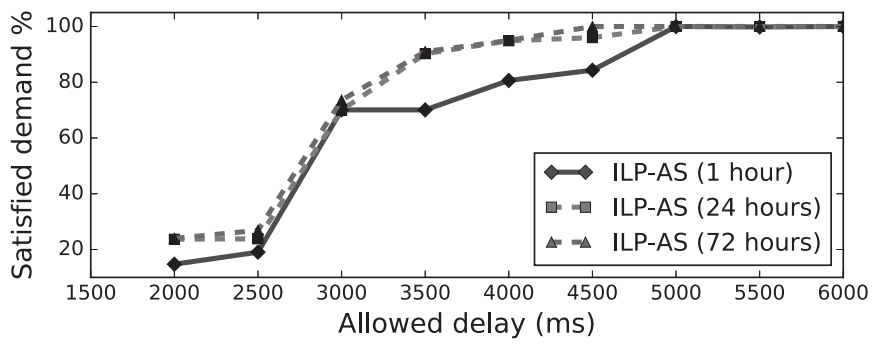

Fig. 5. The current best solution when pausing our ILP-AS solver after 1 hour, 24 hours and 72 hours. We evaluate how much our solution after 1 hour differs from the optimal solution, represented by a 72 hour evaluation. We observe a maximum performance increase of $20 \%$ for a $720 \%$ execution time increase. In this scenario, there are 25 client nodes, 25 services, 2 server and 2 storage nodes in this experiment. $20 \%$ of the service layers are reused by multiple services.

Before we proceed to compare ILP-AS and SPS in a certain time span, we evaluate the influence of the timing where we interrupt the $I L P-A S$ solver. In Fig. 5 we observe the performance of ILP-AS when stopped after 1 hour, after 24 hours and 72 hours. The $\mathrm{X}$-axis represents the allowed response time to satisfy a client. The Y-axis represents the percentage of satisfied clients in the current best solution for a desired response time. There are 25 client nodes, 25 services, 2 server and 2 storage nodes in this experiment. There is always enough storage capacity available to host each service at least once, meaning that we can reach $100 \%$ satisfied demand if the response time tolerance is high enough (increasing $\mathrm{X}$-axis values).

Note that ILP-AS is not able to find a suitable solution for this problem in a feasible execution time without large 
amounts of memory. However, we observe that there is little satisfied demand gained by running the solver for 72 hours compared to only running it for 24 hours. We can assume that running the solver longer will see the same trend. Therefore, we approximate our optimal solution by our 72 hour curve. As such, the satisfied demand in Fig. 5 is not the final solution but only a current best after 1, 24 and 72 hours.

Considering the difference in allowed execution time of the ILP-AS solver, we observe a maximum satisfied demand increase of $20 \%$ for a $720 \%$ execution time increase. For a static environment this may be acceptable but when the demand is rapidly changing, waiting 72 hours may not be feasible. In these environments, using the solution obtained after 1 hour may be more suitable.

Therefore, in the remainder of our simulations we will assume that our solver is interrupted after 1 hour for each run (ILP-AS (1 hour)) and the presented satisfied demand is only a current best solution.

\section{E. Reduced resource consumption}

In this section we compare SPS to our benchmarks, including $I L P-A S$ when the solver is interrupted after 1 hour (ILP-AS (1 hour)). Although ILP-AS would find an optimal solution if given infinite system memory and execution time, by interrupting the solver after 1 hour we can no longer guarantee an optimal solution. Our goal is to show that other methods such as SPS will find a better solution in the same time span due to improved resource consumption. All results were obtained by running a simulation on five different sample topologies with similar characteristics (cfr. Table II) and averaging the results of each run.

Consider Fig. 6, showing the performance of both the ILP$A S$ (1 hour) and SPS approaches, as well as our two other benchmarks $R A$ and $G A$. Once again, the $\mathrm{X}$-axis represents the allowed response time to satisfy a client and the Y-axis represents the percentage of satisfied clients in our current best solution for the desired response time. There are 45 client nodes, 25 services, 2 server and 8 storage nodes in this experiment. The satisfied demand values represent the current best solutions found by interrupting the CPLEX solvers after 1 hour of execution time.

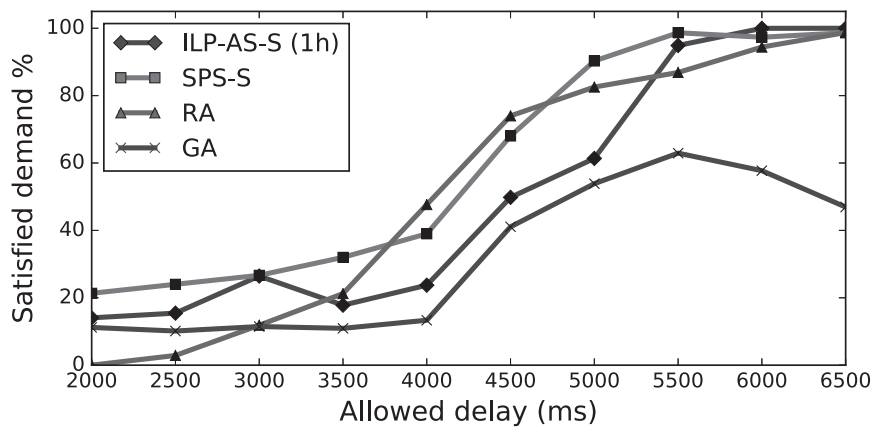

Fig. 6. The satisfied demand for the current best solutions of ILP-AS (1 hour), SPS, RA and GA. There are 45 client nodes, 25 services, 2 server and 8 storage nodes in this experiment. $20 \%$ of the service layers are reused by multiple services. SPS performs best in most scenarios while GA satisfies the least demand.
$G A$ generally satisfies the least users. This is because this algorithm was not made for layered Docker services and expects all layers of a service to be placed on the same storage node. As a result, the services which are placed first will occupy the capacity on nodes in their preferred location, forcing the next services to be placed in suboptimal locations where they cannot serve client requests in time. This is an example of how existing approaches cannot be applied to a Docker approach out of the box, and additional research is required.

Note that in Fig. 6 GA satisfies more clients for 5.5 seconds allowed delay than for 6.5 seconds. This is because $G A$ will choose different storage locations for a service for different amounts of allowed delay (x-axis), always in a greedy manner. In this case, this greedy behavior happened to work out better for the total satisfied demand for 5.5 seconds allowed delay compared to 6 or more seconds. Additional experiments verified that for other simulation parameters (cfr. Table II and Table I), the satisfied demand increased in function of the increasing allowed delay, as expected.

When comparing ILP-AS (1 hour) and SPS, we observe how SPS is generally able to find a solution which satisfies the most users. This is because our dataset in each SPS run is reduced and the solver requires less memory, allowing it to solve the problem faster than ILP-AS. As a result, SPS tends to find better solutions given a limited execution time (e.g. rapidly changing environments). Note that, if the solvers were not interrupted, that eventually ILP-AS would have found the optimal solution.

$R A$ performs very well in the middle of the curve and has the additional benefit that it is easy to implement and executes very quickly. However, when looking at time-sensitive applications with very strict delay requirements (left side $\mathrm{X}$-axis), $R A$ performs the worst. Additionally, when we're looking for the method which can satisfy $100 \%$ of the users with the least delay (right side of X-axis), both ILP-AS (1 hour) and SPS would be better choices than $R A$. For example, in this scenario $S P S$ can satisfy any client request within 5.5 seconds, while $R A$ can only satisfy $85 \%$ of the requests with that delay requirement.

Although none of the approaches aim to minimize the used capacity, it is still interesting to evaluate how each method behaves. Providers could use this evaluation to decide which method is most cost efficient, comparing the required storage capacity with the percentage of satisfied users. Fig. 7 visualizes the used capacity for the same experiments as presented in Fig. 6. We observe how $R A$ uses the same capacity regardless of the allowed delay or satisfied users. This is because $R A$ does not consider the users during the randomized placement steps. As a result, $R A$ is the least cost efficient method when considering storage costs.

$G A$ shows a direct correlation between the satisfied users and the required capacity. This method only uses the capacity that it needs to satisfy users but fails to satisfy a large amount of users compared to the other benchmarks, as previously explained. By grouping all layers of one service on the same storage node, this method fails to make use of the reusability of layers. 


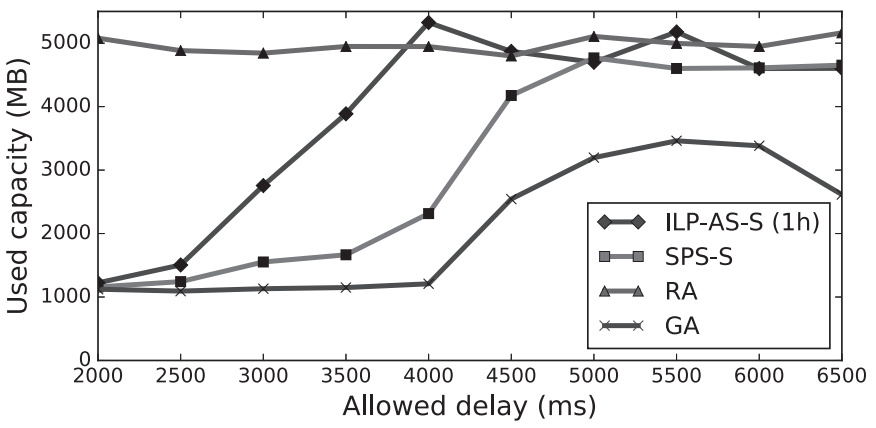

Fig. 7. The corresponding capacity used for the solutions presented in Fig. 6. $R A$ consistently uses the most storage capacity while $G A$ shows a direct correlation between the satisfied users (Fig. 6) and the required storage capacity.

ILP-AS (1 hour) and SPS show a slight correlation between the used capacity and satisfied demand but not nearly as strong as GA. Generally, an increase in satisfied demand will also see an increase in used capacity. This is because in these two approaches, the solver may deploy additional layer replicas to satisfy more clients. However, there are cases where the satisfied demand increases while the used capacity remains the same. This is because the increased allowed delay (X-axis) allows the solver to place layers on more distant storage nodes while still satisfying clients, requiring fewer replicas to be deployed. In the end, both solutions will attempt to maximize the satisfied demand, regardless of the required capacity.

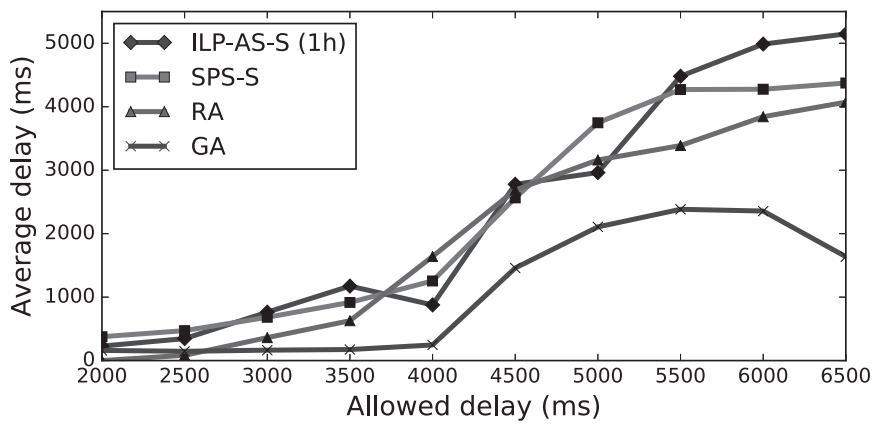

Fig. 8. The average delay for a satisfied client for the solutions presented in Fig. 6. GA shows a direct correlation between the satisfied users (Fig. 6) and the average delay. The remaining methods result in roughly the same delay.

Fig. 8 visualizes the average delay for all satisfied clients in the same experiments as Fig. 6 and Fig. 7. We only display the average delay for the clients who can be served within the allowed delay (X-axis). The less strict our delay requirements become, the higher the average delay is allowed to be while still satisfying the clients. As a result, all curves with the exception of $G A$ follow an increasing average delay trend. $G A$ fails to satisfy clients because it places all layers of a service on the same storage node. This flaw is independent of the allowed delay, which is why we do not see an increase in satisfied demand or average delay when the allowed delay increases.

These simulations show that in rapidly changing environments (short execution times), SPS is able to find a solution that, in most scenarios, satisfies the most demand. Addition- ally, SPS is the second best performing method when it comes to capacity usage, only falling short to $G A$ which in turn also results in the lowest amount of satisfied demand.

In the next section we compare these results with our parallel download model as introduced by Docker v2.0.

\section{F. Serial versus parallel}

Docker v2.0 came with new features which included parallel downloads of all service layers at once, greatly reducing the required time to download a service. If the download time decreases, the importance of the client-server delay increases. In this section we want to evaluate how the parallel download model introduced in Docker v2.0 influences the trends we noticed in the previous section.

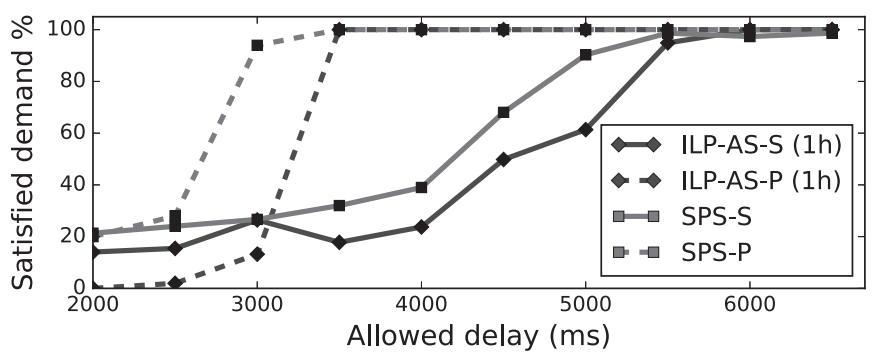

Fig. 9. The satisfied demand in function of the allowed delay for the same experiments as Fig. 6, now comparing ILP-AS-S (1 hour) with ILP-AS-P (1 hour) and $S P S-S$ with $S P S-P$ respectively. We observe that a parallel download model improves the satisfied demand since layers are retrieved quicker.

Consider Fig. 9 where the solid curves (ILP-AS-Sand SPS$S$ ) are the same curves as in Fig. 6, representing the satisfied demand assuming a serial download model. The dashed curves $(I L P-A S-P$ and SPS-P) represent the corresponding satisfied demand for the same experiment when using the parallel problem statement from section IV-B. In a parallel download model we require less time to pull all the layers of a service, so we expect to satisfy more clients than the serial counterpart.

Compare the dashed SPS-P (ILP-AS-P) curve with the solid $S P S-S(I L P-A S-S)$ curve. We observe how the satisfied demand is generally higher for the parallel models, confirming that services in Docker v2 are downloaded faster in parallel and therefore more clients can be satisfied.

An important observation is that for ILP-AS-P the satisfied demand is lower than $I L P-A S-S$ for an allowed delay smaller than three seconds. This is because our solver has more layer placements to consider within the allowed delay when assuming a parallel download model, requiring more execution time to find a solution. Since we interrupt the $I L P-A S-P$ solver after 1 hour, the solver was not able to calculate a good current best solution yet. Note that if we allowed ILP-AS-P to run for more than 1 hour, it would definitely find a solution which satisfies the same or more users than ILP-AS-S, due to the relaxed layer download conditions.

As expected, these results confirm that a parallel download model improves the satisfied demand since layers are retrieved quicker. However, the relaxed delay constraint now results in increased complexity, as more storage nodes can be considered for a suitable placement. 
After comparing the performance of both approaches, we now discuss an interesting use case how this solution can be used to find the minimal required resources for a desired satisfaction level, and thus the minimal cost.

\section{G. Minimum resource requirement}

After evaluating the effect of the execution time for both a serial and parallel download model, we now look at practical usages of our solution. A key objective for any provider is to minimize the cost, often offset against user satisfaction. Our goal is to demonstrate how our research can be used to solve this use case.

Consider Fig. 10, visualizing the satisfied demand (Y-axis) in function of the decreasing storage capacity capacity (Xaxis). There are 45 client nodes, 5 services, 2 server and 2 storage nodes in this experiment. Using this graph, we are able to determine the minimal resources required to achieve a desired satisfaction level. For example, assuming we provision $100 \%$ storage capacity, we can reduce the capacity to $90 \%$ and still maintain $100 \%$ user satisfaction. When using the ILP-AS approach we can even scale down to $80 \%$ capacity and maintain complete satisfaction. This experiment can be repeated and used to avoid overprovisioning.

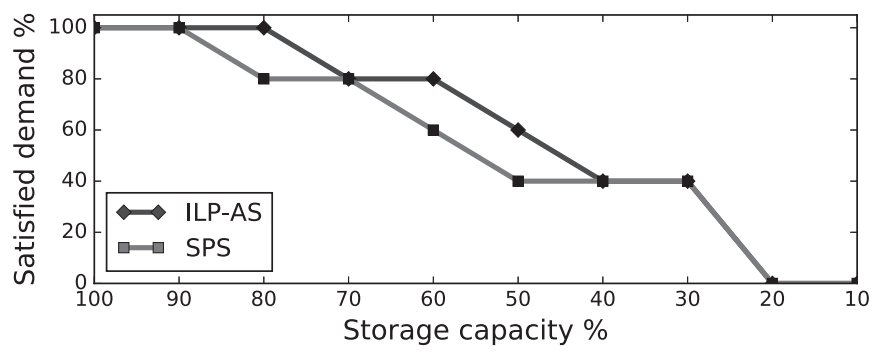

Fig. 10. The importance of the capacity on storage nodes (X-axis) for the satisfied demand (Y-axis). This experiment can be used to determine the minimal resources required to achieve a desired satisfaction level. There are 45 client nodes, 5 services, 2 server and 2 storage nodes in this experiment. $20 \%$ of the service layers are reused by multiple services.

Regardless of the scale of the problem and the desired method, this use case validates that providers are able to use this approach to find minimal resources required for a desired satisfaction level.

\section{CONCLUSION}

In this paper we presented a service placement method using the image layers introduced by Docker. By placing service building blocks called layers in strategic positions, we allow users to connect to a server, retrieve the necessary layers and deploy a service instance. We presented our layer placement problem statement for both a serial and parallel download approach. To reduce the required system resources, we introduce a search heuristic solving the problem one service at a time, considering previous decisions in each step. We evaluate the influence of the sorting order for our search heuristic and provide four different sorting methods. Simulation results show that the iterative search method finds a better solution in the same time span due to improved resource consumption. We explain how we obtained our simulation results which are based on real-life data obtained from crawling the Docker repositories and modeling real network distributions. Last, we introduce an interesting use-case to find the minimal storage resources necessary for a desired satisfaction level, avoiding any overprovisioning.

As conclusion we can say that our model is able to find a suitable layer placement to maximize the satisfied demand, using an on-demand deployment scheme. By reusing previously deployed layers, we avoid cluttering the network with layer replicas.

In future work we look at dynamic placement algorithms to adjust to the dynamic service demand. With our current method we are able to find the best placement for initial demand but changes are necessary when the available session slots on servers are occupied. We will develop placement and selection algorithms using frequent reports from servers and storages as input. This allows us to place data where it is most needed by analyzing demand patterns. We can also use this data to auto-scale server and storage resources based on the changing demand, optimizing the amount of clients we can serve without overprovisioning. Additionally, we will build larger evaluation scenarios and use emulation techniques [34] to evaluate our solutions. Recently, a number of frameworks have been presented in literature [35] for container orchestration on a cluster of low-cost devices such as Raspberry Pi. These platforms provide an excellent tool to evaluate our solutions in more realistic conditions.

\section{ACKNOWLEDGEMENTS}

This project was partly funded by the UGent BOF-GOA project "Autonomic Networked Multimedia Systems".

\section{REFERENCES}

[1] M. Satyanarayanan, P. Bahl, R. Caceres, and N. Davies, "The case for vm-based cloudlets in mobile computing," IEEE Pervasive Computing, vol. 8, no. 4, pp. 14-23, Oct 2009.

[2] J. Thönes, "Microservices," IEEE Software, vol. 32, no. 1, pp. 116-116, Jan 2015.

[3] N. Dragoni, S. Giallorenzo, A. Lluch-Lafuente, M. Mazzara, F. Montesi, R. Mustafin, and L. Safina, "Microservices: yesterday, today, and tomorrow," CoRR, vol. abs/1606.04036, 2016. [Online]. Available: http://arxiv.org/abs/1606.04036

[4] C. Costache, O. Machidon, A. Mladin, F. Sandu, and R. Bocu, "Software-defined networking of linux containers," in 2014 RoEduNet Conference 13th Edition: Networking in Education and Research Joint Event RENAM 8th Conference, Sept 2014, pp. 1-4.

[5] R. Morabito, V. Cozzolino, A. Y. Ding, N. Beijar, and J. Ott, "Consolidate iot edge computing with lightweight virtualization," IEEE Network, vol. 32, no. 1, pp. 102-111, Jan 2018.

[6] C. Anderson, "The long tail," Wired magazine, vol. 12, no. 10, pp. 170$177,2004$.

[7] A. Ojala and P. Tyrvainen, "Developing cloud business models: A case study on cloud gaming," IEEE Software, vol. 28, no. 4, pp. 42-47, July 2011.

[8] C. Weinhardt, A. Anandasivam, B. Blau, N. Borissov, T. Meinl, W. Michalk, and J. Stößer, "Cloud computing - a classification, business models, and research directions," Business \& Information Systems Engineering, vol. 1, no. 5, pp. 391-399, Oct 2009. [Online]. Available: https://doi.org/10.1007/s12599-009-0071-2

[9] C. Anderson, "Docker [software engineering]," IEEE Software, vol. 32, no. 3, pp. 102-c3, May 2015. 
[10] P. Smet, B. Dhoedt, and P. Simoens, "On-demand provisioning of longtail services in distributed clouds," in NOMS 2016 - 2016 IEEE/IFIP Network Operations and Management Symposium, April 2016, pp. $1320-1323$.

[11] A. Amiri, "Application placement and backup service in computer clustering in software as a service (saas) networks," Computers \& Operations Research, vol. 69, pp. 48 - 55, 2016. [Online]. Available: http://www.sciencedirect.com/science/article/pii/S030505481500283X

[12] Q. Zhang, Q. Zhu, M. Zhani, R. Boutaba, and J. Hellerstein, "Dynamic service placement in geographically distributed clouds," Selected Areas in Communications, IEEE Journal on, vol. 31, no. 12, pp. 762-772, December 2013

[13] J. Famaey, T. Wauters, F. De Turck, B. Dhoedt, and P. Demeester, "Network-aware service placement and selection algorithms on largescale overlay networks," Computer Communications, vol. 34, no. 15, pp. 1777-1787, 2011.

[14] M. Selimi, D. Vega, F. Freitag, and L. Veiga, "Towards network-aware service placement in community network micro-clouds," in European Conference on Parallel Processing. Springer, 2016, pp. 376-388.

[15] M. Selimi, L. Cerdà-Alabern, M. Sánchez-Artigas, F. Freitag, and L. Veiga, "Practical service placement approach for microservices architecture," in Proceedings of the 17th IEEE/ACM International Symposium on Cluster, Cloud and Grid Computing, ser. CCGrid '17. Piscataway, NJ, USA: IEEE Press, 2017, pp. 401-410. [Online]. Available: https://doi.org/10.1109/CCGRID.2017.28

[16] W. Tärneberg, A. Mehta, E. Wadbro, J. Tordsson, J. Eker, M. Kihl, and E. Elmroth, "Dynamic application placement in the mobile cloud network," Future Generation Computer Systems, vol. 70, pp. 163 - 177, 2017. [Online]. Available: http://www.sciencedirect.com/science/article/pii/S0167739X16302060

[17] J. K. Yeoh and D. K. Chua, "Optimizing crane selection and location for multistage construction using a four-dimensional set cover approach," Journal of Construction Engineering and Management, vol. 143, no. 8, p. 04017029, 2017.

[18] P. Bahrampour, M. Safari, and M. B. Taraghdari, "Modeling multiproduct multi-stage supply chain network design," Procedia Economics and Finance, vol. 36, pp. 70-80, 2016.

[19] T. Wu, F. Chu, Z. Yang, and Z. Zhou, "A lagrangean relaxation approach for a two-stage capacitated facility location problem with choice of depot size," in Networking, Sensing and Control (ICNSC), 2015 IEEE 12th International Conference on, April 2015, pp. 39-44.

[20] J. Li, F. Chu, C. Prins, and Z. Zhu, "Lower and upper bounds for a two-stage capacitated facility location problem with handling costs," European Journal of Operational Research, vol. 236, no. 3, pp. 957-967, 2014.

[21] B. Gendron, P.-V. Khuong, and F. Semet, "Comparison of formulations for the two-level uncapacitated facility location problem with single assignment constraints," Computers \& Operations Research, vol. 86, pp. 86 - 93, 2017. [Online]. Available: http://www.sciencedirect.com/science/article/pii/S0305054817300540

[22] P. Bellavista and A. Zanni, "Feasibility of fog computing deployment based on docker containerization over raspberrypi," in Proceedings of the 18th International Conference on Distributed Computing and Networking, ser. ICDCN '17. New York, NY, USA: ACM, 2017, pp. 16:1-16:10. [Online]. Available: http://doi.acm.org/10.1145/3007748.3007777

[23] C. Dupont, R. Giaffreda, and L. Capra, "Edge computing in iot context: Horizontal and vertical linux container migration," in 2017 Global Internet of Things Summit (GIoTS), June 2017, pp. 1-4.

[24] O. Skarlat, M. Nardelli, S. Schulte, and S. Dustdar, "Towards qos-aware fog service placement," in 2017 IEEE 1st International Conference on Fog and Edge Computing (ICFEC), May 2017, pp. 89-96.

[25] M. I. Naas, P. R. Parvedy, J. Boukhobza, and L. Lemarchand, "ifogstor: An iot data placement strategy for fog infrastructure," in 2017 IEEE 1st International Conference on Fog and Edge Computing (ICFEC), May 2017, pp. 97-104.

[26] R. S. Garfinkel and G. L. Nemhauser, Integer programming. Wiley New York, 1972, vol. 4.

[27] J. Famaey, T. Wauters, F. De Turck, B. Dhoedt, and P. Demeester, "Towards efficient service placement and server selection for large-scale deployments," in Telecommunications, 2008. AICT'08. Fourth Advanced International Conference on. IEEE, 2008, pp. 13-18.

[28] (2013) ilab.t virtual wall | internet based communication networks and services. [Online]. Available: http://www.ibcn.intec.ugent.be/content/ilabt-virtual-wall

[29] (2017) Docker hub. Docker Inc. [Online]. Available: https://hub.docker.com/
[30] F. Lobillo Vilela, A. Juan Ferrer, M. A. Puente, Z. Becvar, M. Rohlik, T. Vanek, P. Mach, O. Muñoz Medina, J. Vidal Manzano, H. Hariyanto et al., "Tropic-d22 design of network architecture for femto-cloud computing," 2013

[31] How fast is $4 \mathrm{~g}$ ? $4 \mathrm{~g}$ speeds and uk network performance. [Online]. Available: http://www.4g.co.uk/how-fast-is-4g/

[32] A. Jain and J. Pasquale, "Internet distance prediction using node-pair geography," in Network Computing and Applications (NCA), 2012 11th IEEE International Symposium on. IEEE, 2012, pp. 71-78.

[33] I. SoftLayer Technologies. Softlayer ip backbone :: Looking glass. [Online]. Available: http://lg.softlayer.com/

[34] A. Lertsinsrubtavee, A. Ali, C. Molina-Jimenez, A. Sathiaseelan, and J. Crowcroft, "Picasso: A lightweight edge computing platform," in 2017 IEEE 6th International Conference on Cloud Networking (CloudNet), Sept 2017, pp. 1-7.

[35] L. Miori, J. Sanin, and S. Helmer, "A platform for edge computing based on raspberry pi clusters," in Data Analytics, A. Calì, P. Wood, N. Martin, and A. Poulovassilis, Eds. Cham: Springer International Publishing, 2017, pp. 153-159.

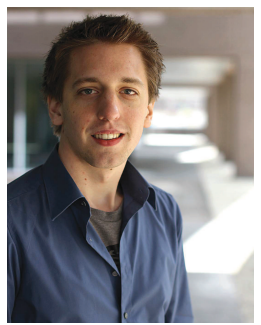

Piet Smet received his M.Sc degree in Informatics (2012) from Hogeschool Ghent, Belgium. He wrote a thesis on the development of a large-data social media game during his Masters degree. In August 2012 he started a Ph.D. degree on 'Service-Centric Networking' at Ghent University, supervised by B.Dhoedt and P. Simoens. Piet Smet was also active as a researcher on the European research project H2020 FUSION.

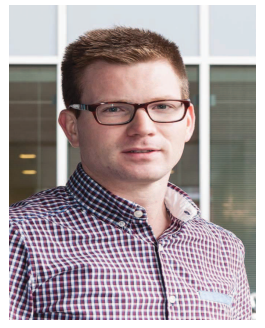

Pieter Simoens received his M.Sc. degree in Electronic Engineering (2005) and Ph.D. degree (2011) from the Ghent University, Belgium. During his $\mathrm{Ph} . \mathrm{D}$. research, he was funded by the Fund for Scientific Research Flanders (FWO-V). In 2012, he was a visiting researcher at the School of Computer Science of Carnegie Mellon University, USA. Currently, he is assistant professor affiliated with the Department of Information Technology of the Ghent University and with iMinds. His main research interests include mobile cloud offloading, serviceoriented networking, edge/fog computing paradigms, and service engineering for advanced mobile applications. In these fields, he is author and co-author of more than 70 papers published in international journals or in the proceedings of international conferences. He has also been involved in several national and European research projects (FP6 MUSE, FP7 MobiThin, H2020 FUSION).

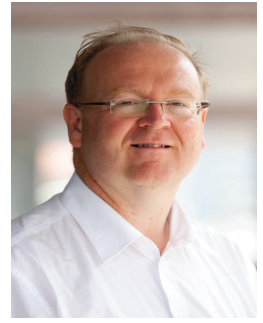

Bart Dhoedt received a Masters degree in Electrotechnical Engineering (1990) from Ghent University. His research, addressing the use of micro-optics to realize parallel free space optical interconnects, resulted in a Ph.D. degree in 1995. After a 2-year post-doc in opto-electronics, he became Professor at the Department of Information Technology. He is author or co-author of more than 300 publications in international journals or conference proceedings. 